\title{
Redrawn Phase-Separated Borosilicate Glasses: A TEM Investigation
}

\author{
Stefano Polizzi $\left({ }^{1}\right)$, Aldo Armigliato $\left({ }^{2}\right)$, Pietro Riello $\left({ }^{1}\right)$, \\ Nicholas F. Borrelli $\left({ }^{3}\right)$ and Giuliano Fagherazzi $\left({ }^{1}\right)$ \\ $\left({ }^{1}\right)$ Dipartimento di Chimica Fisica, Università di Venezia, Dorsoduro 2137, 30123 Venezia, Italy \\ $\left({ }^{2}\right)$ CNR, Instituto LAMEL, via Gobetti 101, 40129 Bologna, Italy \\ $\left({ }^{3}\right)$ Corning Glass Research and Development, Corning, New York 14831, USA
}

(Received February 17; accepted May 22, 1997)

\author{
PACS.61.16.Bg - Transmission, reflection and scanning electron microscopy (including EBIC) \\ PACS.61.43.Fs - Glasses \\ PACS.81.40.-z - Treatment of materials and its effects on microstructure and properties
}

\begin{abstract}
Two alkali aluminoborosilicate glasses containing oriented crystalline particles have been investigated by Transmission Electron Microscopy (TEM). Both materials have been thermally treated in order to phase-separate crystalline droplets and have been then redrawn at a temperature above their softening point to elongate and orient them. In one sample the crystalline phase is a $\mathrm{Ag}(\mathrm{Cl}, \mathrm{Br})$ solid solution, which gives the material birefringence properties, while in the other sample the particles on the surface have been chemically reduced to $\mathrm{Ag}$, giving the material polarizing properties (Polarcor ${ }^{\mathrm{TM}}$ ). Most of the observed needle-like particles are built up by a small number of well grown crystallites often separated by voids. Both the crystallites and the voids occupy the whole width of the particles and can assume very different lengths. Crystallites inside a particle have different orientations.
\end{abstract}

\section{Introduction}

In a previous paper [1] we studied by Small Angle X-ray Scattering (SAXS) two borosilicate glasses in which a metal halide crystalline phase is thermally developed in a controlled way. Depending on the preparation conditions this class of materials have different optical properties which have been exploited in a-number of products, ranging from photochromic eyeglasses to polarizers for optical devices such as CD players [2-7]. In particular, both materials studied in the previous paper underwent a redrawn process at a temperature above their softening point and one was also chemically reduced.

The use of Transmission Electron Microscopy (TEM) in redrawn samples $[6,7]$ indicated the formation of elongated crystalline particles very well oriented along the stretching direction. In order to improve the determination of particle dimensions by using a volume averaging technique, in the previous paper we used 2D-Small Angle X-ray Scattering (SAXS) to study both a birefringent and a polarizing sample (obtained by reduction of the crystalline phase). The data were analyzed by a two-dimensional fit of a suitable model of oriented prolate ellipsoids, and particle distributions with an average size of $22 \mathrm{~nm} \times 370 \mathrm{~nm}$ in the birefringent material 


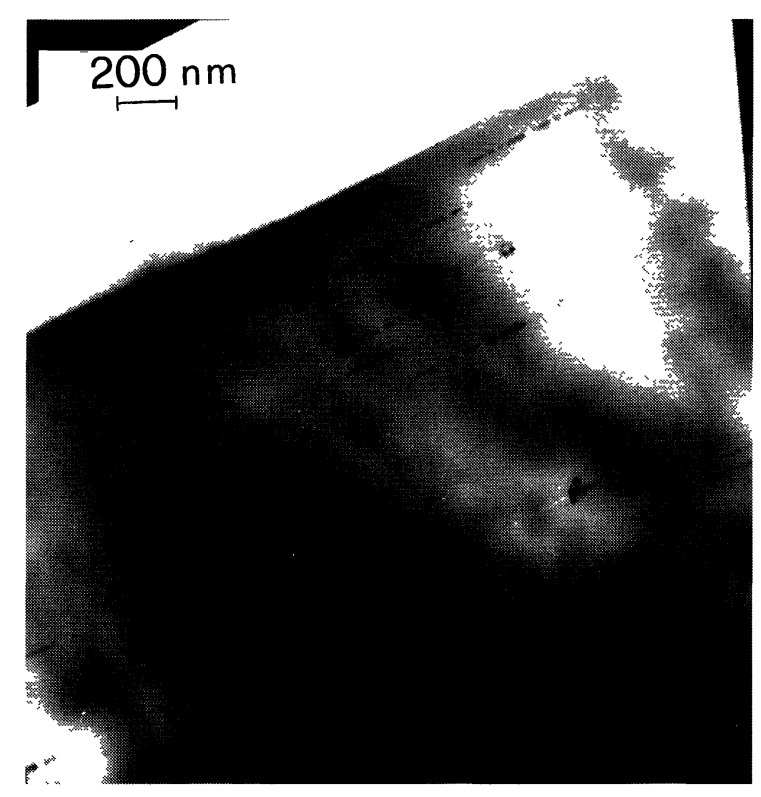

Fig. 1. - Cross-sectional TEM image of sample I. The surface of the sample is visible on the top of the image.

and $18 \mathrm{~nm} \times 230 \mathrm{~nm}$ in the polarizing glass have been obtained. The length distributions are skewed up to very high length values. In the reduced sample a narrow distribution of much shorter particles $(14 \mathrm{~nm} \times 30 \mathrm{~nm})$ was also found, whose nature was puzzling. In the same paper, line broadening analysis of X-ray Diffraction (XRD) profiles indicated crystallites of $14 \mathrm{~nm}$ average size for the birefringent sample and $10 \mathrm{~nm}$ for the polarizing one.

In the present study we have used TEM with microanalytical capabilities on the same two samples, in order to investigate the internal morphology and structure of the elongated particles and in the attempt to identify the population of short particles in the polarizing sample.

\section{Experimental}

\subsection{Samples}

The starting glass belongs to a class of well studied photochromic glasses [8]. Its composition in wt.\% is as follows: $\mathrm{SiO}_{2}(56), \mathrm{B}_{2} \mathrm{O}_{3}(18), \mathrm{Al}_{2} \mathrm{O}_{3}(6), \mathrm{K}_{2} \mathrm{O}(6), \mathrm{ZrO}_{2}$ (5), $\mathrm{Na}_{2} \mathrm{O}$ (4), $\mathrm{Li}_{2} \mathrm{O}(2)$, $\mathrm{Ag}(0.21), \mathrm{Cl}(0.17), \mathrm{Br}(0.14), \mathrm{CuO}(0.006)$. Samples are obtained from glass slabs of high optical quality, cut, ground and polished. Two samples have been studied: sample I has been preliminary heat treated at about $720^{\circ} \mathrm{C}$ and then drawn under an applied stress of $34.5 \mathrm{MPa}$ at a temperature above the softening point; sample II has undergone a similar treatment and has been then chemically reduced in hydrogen at $430{ }^{\circ} \mathrm{C}$. The samples thickness was about $0.86 \mathrm{~mm}$ and the reduced layer in sample II was about $20 \mu \mathrm{m}$ on both faces of the platelet, as determined by optical microscopy. 

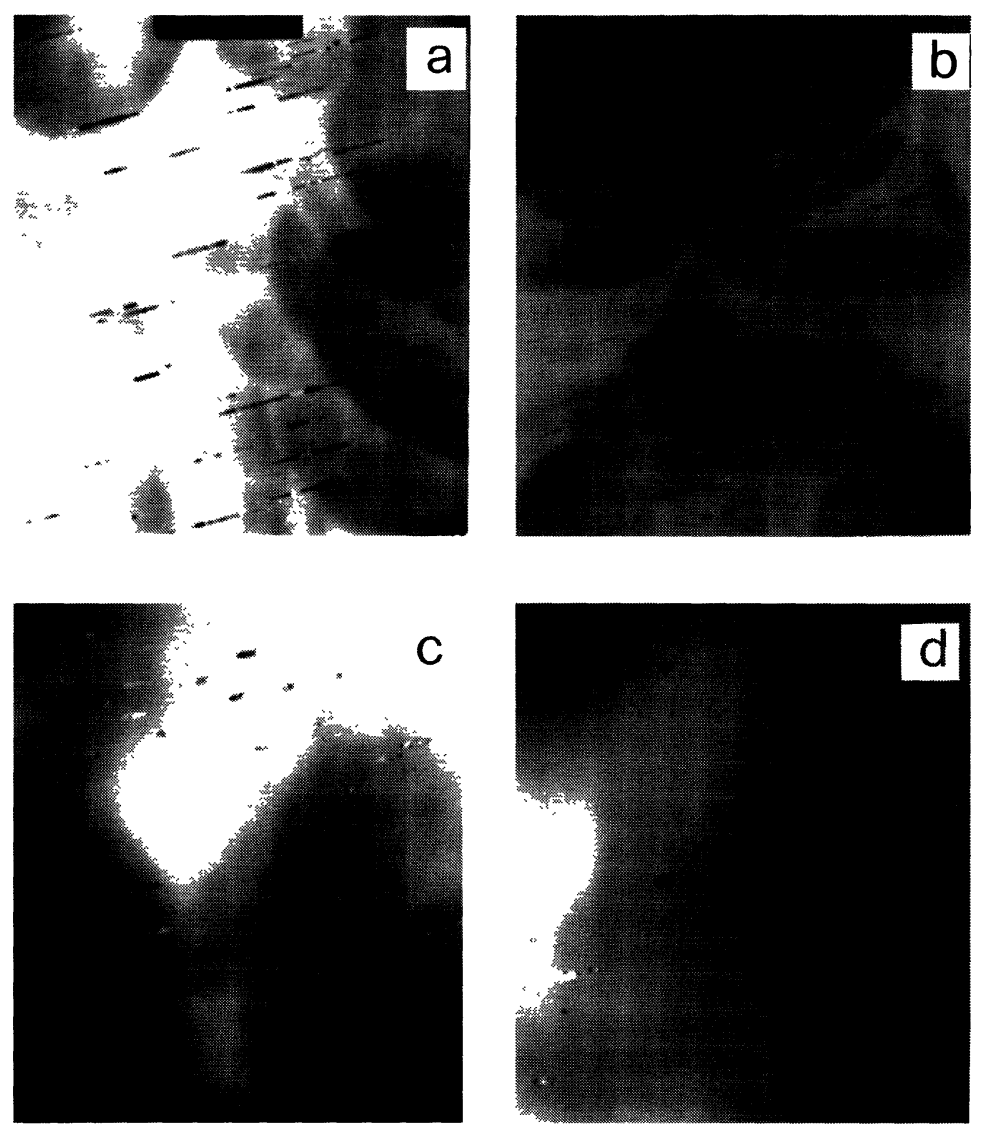

\section{$200 \mathrm{~nm}$}

Fig. 2. - Cross-sectional TEM micrographs taken at different depths $z$ from the surface in sample I: a) $50 \mu \mathrm{m}$; b) $100 \mu \mathrm{m}$; ) $150 \mu \mathrm{m}$; d) $165 \mu \mathrm{m}$.

\subsection{TEM}

Samples for the electron microscopy observations were prepared as cross sections by a standard procedure involving cutting, gluing and lapping down to $20 \mu \mathrm{m}$, followed by a final thinning step in a Gatan DuoMill ion beam miller [9]. Samples I and II were glued face to face in the same sandwich, in order to image areas of comparable thickness of the two samples in a single picture.

The TEM employed was a Philips CM30, operating at $300 \mathrm{kV}$. It is equipped with an EDAX $9900 \mathrm{X}$-ray microanalyzer, that was employed to determine the local composition of the sample. 


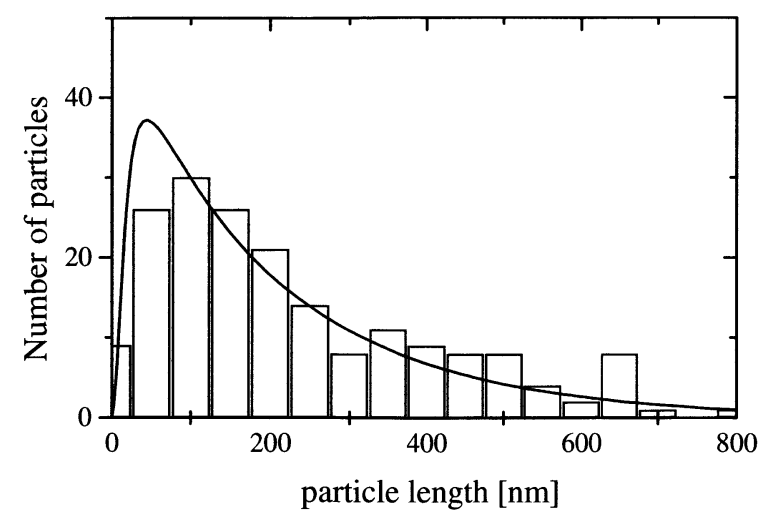

Fig. 3. - Histogram of the particle lengths measured on a large number of TEM images of sample I (also at different depths) and the distribution obtained by SAXS in a previous work on the same sample.

\section{Results and Discussion}

\subsection{Sample I}

Figure 1 shows a cross-sectional TEM micrograph of a region just below the surface of the sample. The elongated $\mathrm{Ag}(\mathrm{Cl}, \mathrm{Br})$ particles are randomly distributed in the glass matrix, but with their long axes very well oriented along the stretching direction. Furthermore, a complicated inner structure is apparent. Figures 2a-d show micrographs taken at different depths from the surface. First of all, it is evident that the sample is not homogeneous along its depth: within the first $100 \mu \mathrm{m}$ the distribution of particle dimensions seems to be more or less constant but after that, particles become shorter and shorter; at $165 \mu \mathrm{m}$ they are already egg-like and in the core of the sample they are presumably spherical. The whole sample depth being about $860 \mu \mathrm{m}$, this means that only one third of the sample has been affected by the stretching. This inhomogeneity is likely to be due to a temperature gradient along the glass slab depth, occurred during the redrawing process: a lower temperature means a lower viscosity and smaller attainable elongation values.

Figure 3 shows the histogram obtained by measuring the particle length in a number of micrographs at different depths. This histogram is in very good agreement with the distribution of particles obtained by SAXS on the same sample [1], also shown for comparison.

Looking at the inner structure of the particles it can be seen that most of them are built up by a small number of regions with different contrast; these regions usually fill up the width of the particle and their length ranges from very small (few $\mathrm{nm}$ ) to something like one third of the whole particle. The crystallite size obtained in the previous paper by XRD line profile analysis looks like an average value of all these dimensions. Only a small amount of particles has a more complicated structure, showing dark points scattered inside the outline of a particle. In order to study the nature of the inner regions, the properties of the diffraction contrast imaging mode have been exploited; namely, the sample has been tilted and changes of the contrast have been followed. Figure 4 shows a typical situation: it is evident that the contrast depends on the orientation, which indicates that the originally brighter regions are crystallites differently oriented with respect to the darker ones. Nevertheless, not all the bright regions are seen to change their contrast, hence they could be voids present inside the particle. To check that, 

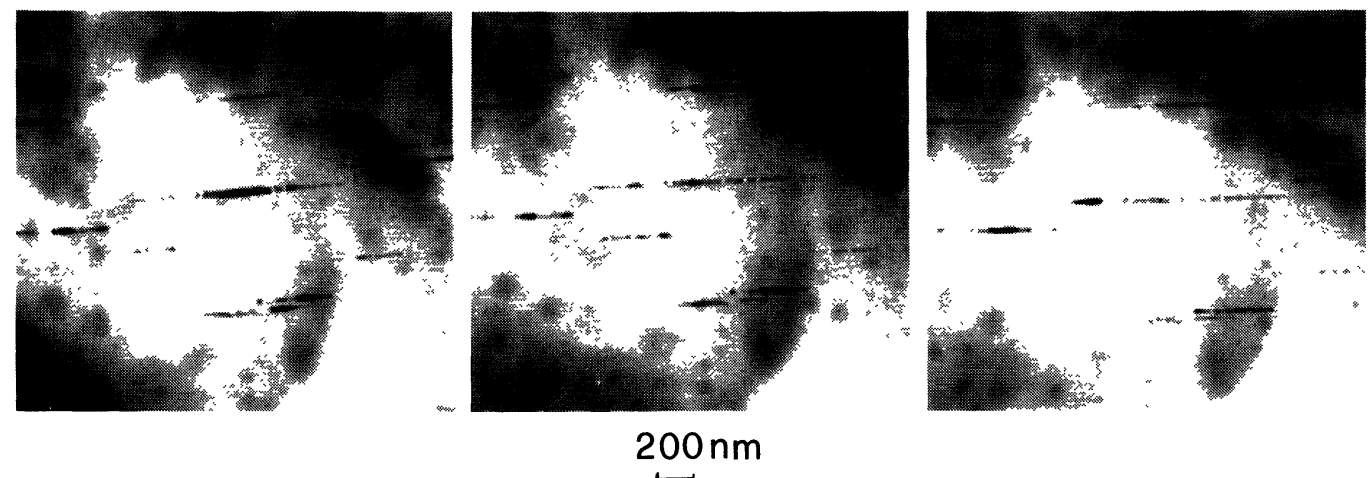

$200 \mathrm{~nm}$

Fig. 4. - Same area of sample I taken at three different tilting angles.

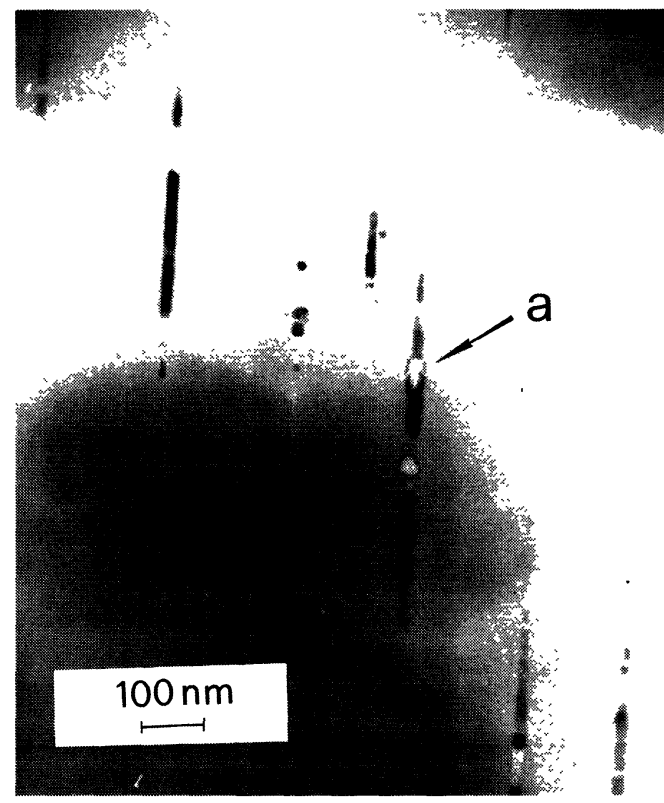

Fig. 5. - Area of sample I where microanalysis has been performed. The arrows indicate the small "dark" (a) and "bright" (b) area where the beam has impinged, destroying that part of the particle.

different regions of single particles have been investigated by X-ray microanalysis. Figure 5 shows the "dark" and "bright" regions analyzed: since the high density microbeam destroys the glass locally, the investigated regions are directly recognizable. In Figure 6 the relevant spectra are shown: besides the matrix elements, $\mathrm{Ag}, \mathrm{Cl}, \mathrm{Br}$ have been detected in the dark region, while the absence of the latter elements in the bright region identifies it as a void. These voids seem to be present in most particles regardless of their length and are likely produced during the redrawing process and/or during the following cooling phase. In fact, the silver halide phase is in the liquid state during stretching [10] and will occupy a smaller volume after solidification. Also, some void could be formed upon slip between glass and silver halide during stretching. 


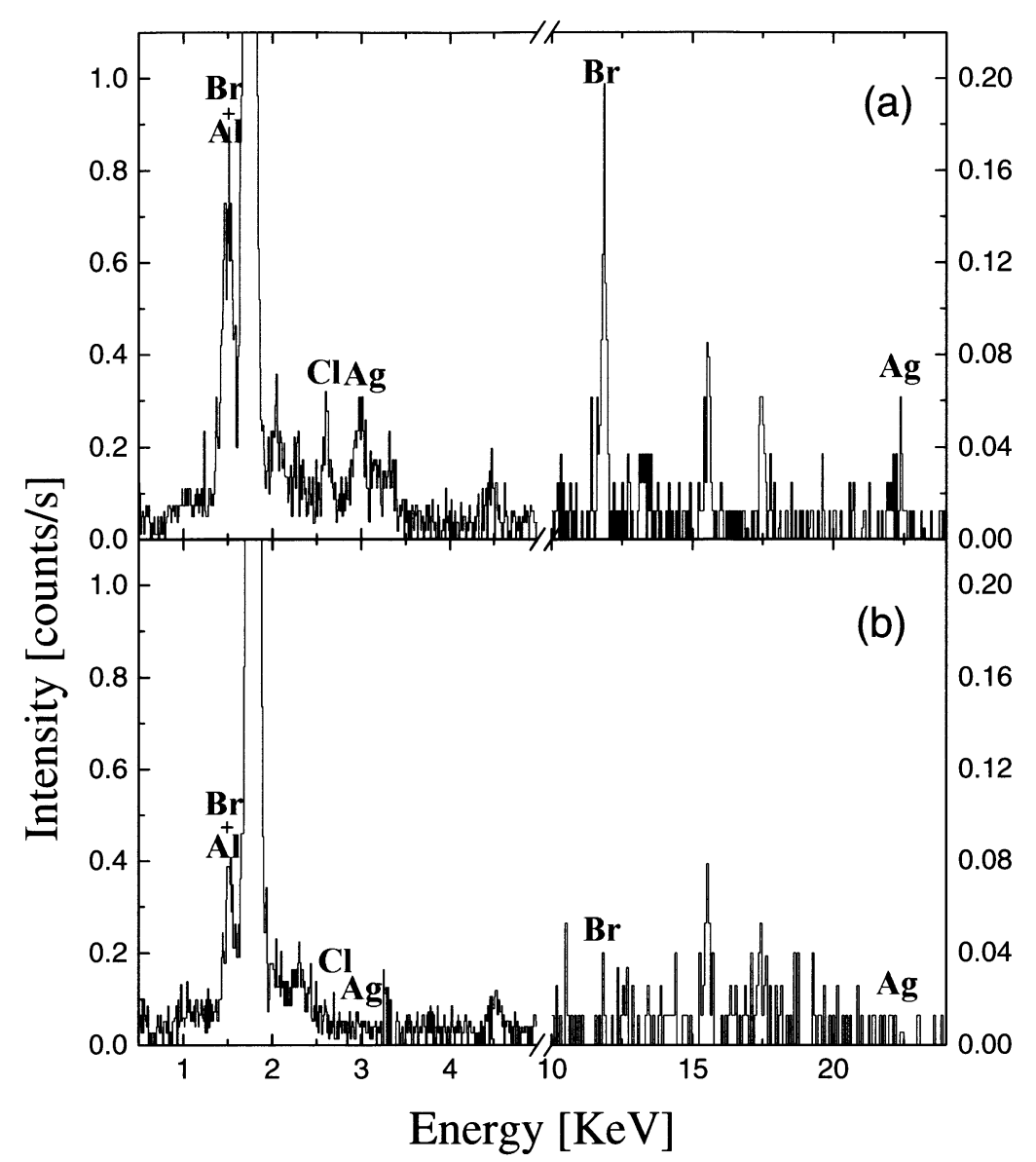

Fig. 6. - Microanalysis of the two areas labeled (a) and (b) in Figure 5. The non-labeled peaks are due to other components of the glass or to the sample holder.

In the inset of Figure 7 the microdiffraction of the dark region indicated in the same figure is shown. The observed spots are indexed and correspond to the [120] crystallographic orientation of the cubic $\mathrm{Ag}(\mathrm{Cl}, \mathrm{Br})$ solid solution (space group: $\mathrm{Fm} \overline{3} \mathrm{~m})$. The interplanar spacings are in agreement with the lattice constant value of $a_{0}=0.5696(5) \mathrm{nm}$ determined by XRD in a previous paper [1]. In this particular case, the crystallite seems to be approximately oriented with the $\overline{8} 24$ crystallographic direction oriented in the stretching direction, but different orientations have been found in other crystals.

\subsection{Sample II}

First of all, it catches one's eye (see Figs. 8 and 9) that the number density of particles is much higher than in sample I, which was not evident in our previous SAXS analysis. For this sample the investigation along the depth of the platelet has not evidenced any significant difference in the particle dimensions. Even a possible difference between the reduced surface layer (the first $20 \mu \mathrm{m}$ ) and the rest of the sample, where the reduction should not have taken place (bulk), is difficult to appraise. Figure 8 shows a typical image of particles close 


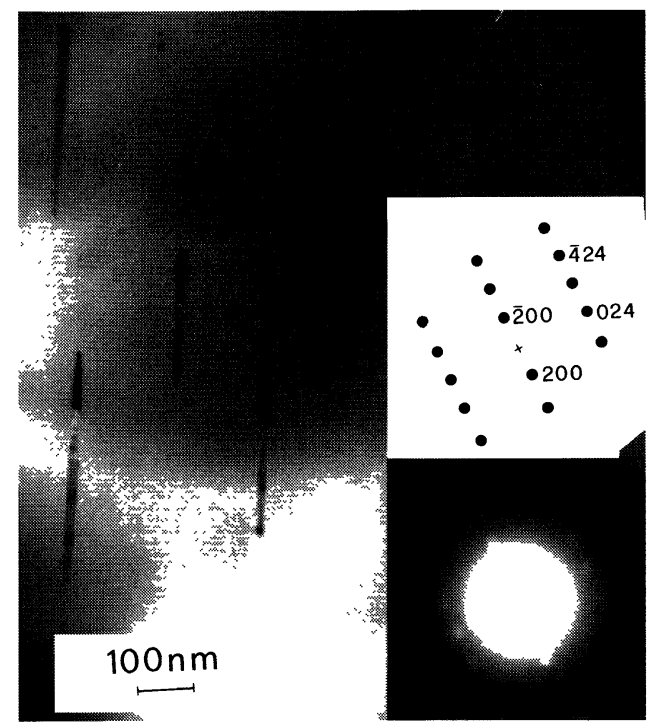

Fig. 7. - Area of sample I where microdiffraction has been carried out. The diffraction pattern in the inset refers to the "dark" part of a particle. Some crystallographic directions of the cubic $\mathrm{Ag}(\mathrm{Cl}$, $\mathrm{Br}$ ) solid solution are shown (200 and spots have been overexposed to make the weaker spots visible).

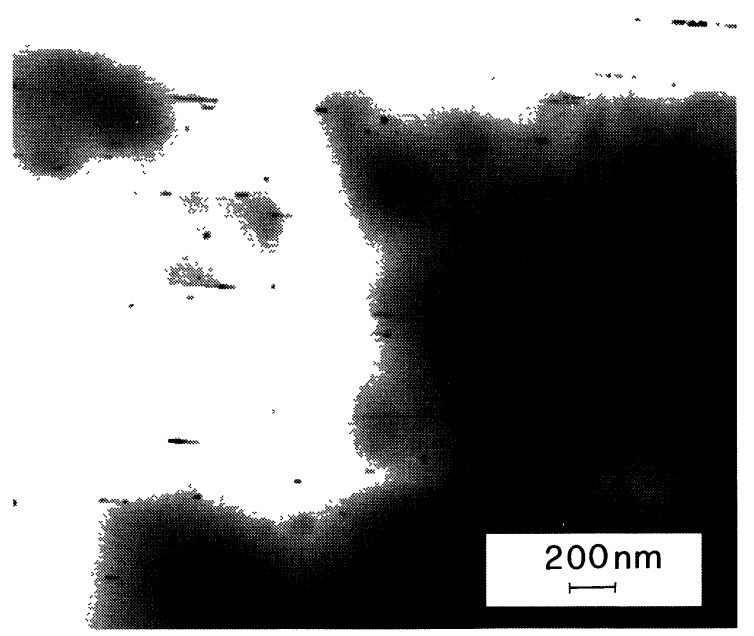

Fig. 8. - Cross-sectional TEM image of sample II. The surface of the sample is visible on the top of the image.

to the surface and Figure 9 shows particles in the bulk $(z=40 \mu \mathrm{m})$. The reduced layer seems to contain a larger number of very dark small regions, mainly concentrated at the particle ends. It would be tempting to identify these regions with the population of small particles (average $14 \mathrm{~nm} \times 30 \mathrm{~nm}$ ) revealed by our previous SAXS analysis [1]. It could be argued that the reduced surface layer, which is less than $5 \%$ of the whole sample, should not contribute so strongly to the SAXS intensity, but it must be kept in mind that SAXS is proportional 


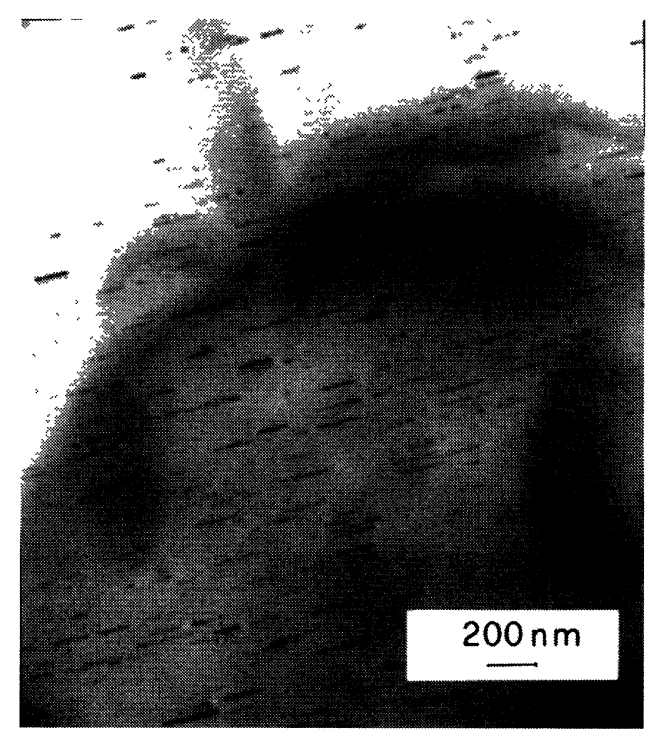

Fig. 9. - Cross-sectional TEM image of sample II taken in the un-reduced part of the sample $(z=40 \mu \mathrm{m})$.

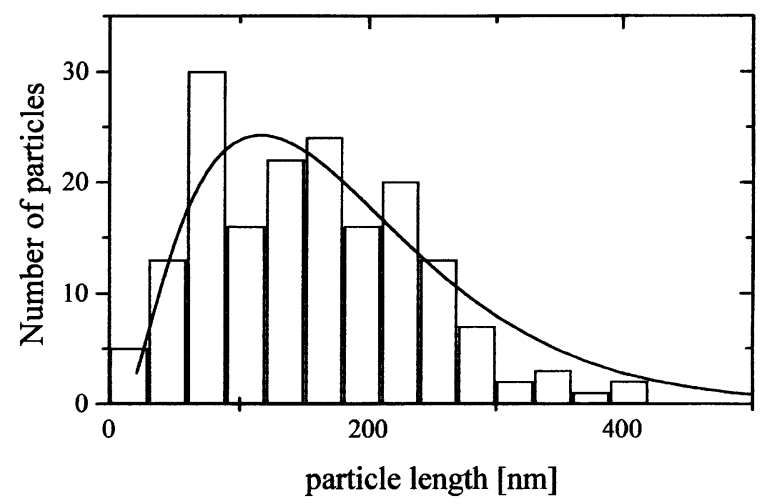

Fig. 10. - Histogram of the particle lengths measured on a large number of TEM images of sample II (also at different depths) and the distribution obtained by SAXS in a previous work on the same sample.

to the electron density difference which is higher for Ag particles on the surface than for the $\mathrm{Ag}(\mathrm{Cl}, \mathrm{Br})$ ones in the bulk. Anyway, on the ground of the present data this is little more than a speculation, which needs further confirmations.

In Figure 10 the histogram obtained from the different TEM images of sample II is shown together with the distribution of larger particles obtained by SAXS on the same sample [1]. Unfortunately, the images do not allow us to unambiguously identify small particles to be compared with the second distribution obtained in that paper.

Microanalysis performed on a few particles of this sample have shown a similar inner structure of the particles as in sample I. Of course the particles close to the surface did not show any presence of $\mathrm{Cl}$ and $\mathrm{Br}$, having been reduced to $\mathrm{Ag}$ by the $\mathrm{H}_{2}$ exposure. 


\section{Conclusions}

The use of the TEM microanalytical capabilities together with diffraction imaging mode has allowed us to clarify the inner structure of the elongated particles present in these polarizing materials. On the contrary, the morphological changes induced by the reduction process and the nature of the population of very small particles are still unclear and need further investigations.

\section{Acknowledgments}

The authors are indebted to A. Garulli and F. Corticelli for their skillful technical assistance.

\section{References}

[1] Polizzi S., Riello P., Fagherazzi G., Bark, M. and Borrelli N.F., J. Appl. Cryst. 30 (1997) 487.

[2] Armistead W.H. and Stookey S.D., Science 144 (1964) 150.

[3] Araujo R.J., Photochromic Glass, Treatise on Material Science and Technology, M. Tomozawa and R.H. Doremus, Eds., vol. 12 (Academic Press, New York, 1977).

[4] Seward T.P. III, SPIE-Proc. 464 (1984) 96.

[5] Borrelli N.F. and Seward T.P. III, in "Engineered Materials Handbook", vol. 4 (ASM International, Ohio, 1992) pp. 439-444.

[6] Borrelli N.F. and Davis C.L., SPIE-Proc. 1746 (1992) 336.

[7] Borrelli N.F., SPIE-Proc. 1761 (1992) 202.

[8] Borrelli N.F. and Wedding B., J. Appl. Phys. 63 (1988) 2756.

[9] Garulli A., Armigliato A. and Vanzi M., J. Microsc. Spectrosc. Electron 10 (1985) 135.

[10] Wedding, B., Ceram. Bull. 56 (1977) 334. 